\title{
Assisting the Elite-Driven Open Source Development through Activity Data
}

\author{
Zhendong Wang \\ zhendow@uci.edu \\ University of California, Irvine \\ California, USA
}

\begin{abstract}
Elite developers, who own the administrative privileges for a project, maintain a diverse profile of contributing activities, and drive the development of open source software (OSS). To advance our understanding and further support the OSS community, I present a fresh approach to investigate developers' public activities from the fine-grained event data provided by GiтHuв. Further, I develop this approach into an analysis framework for collecting, modeling, and analyzing elite developers' online contributing activities. Employing this framework, I have conducted empirical studies on various OSS projects and ecosystems to characterize elite developers' full-spectrum activities and their dynamics, and also unveil relationships between their effort allocation and projects' technical outcomes. Finally, I propose to design and implement a toolset based on this framework and my results to date, which supports individual developers' decision-making and assists their routine workflows with automation.
\end{abstract}

\section{CCS CONCEPTS}

- Software and its engineering $\rightarrow$ Collaboration in software development; Open source model.

\section{KEYWORDS}

Elite developers, open source software, project outcomes

\section{ACM Reference Format:}

Zhendong Wang. 2020. Assisting the Elite-Driven Open Source Development through Activity Data. In Proceedings of the 28th ACM Joint European Software Engineering Conference and Symposium on the Foundations of Software Engineering (ESEC/FSE '20), November 8-13, 2020, Virtual Event, USA. ACM, New York, NY, USA, 4 pages. https://doi.org/10.1145/3368089.3418541

\section{INTRODUCTION}

The rapid development of open source software (OSS) has become one of the major engines for software innovation, and it receives tremendous attention from academia and industry. Particularly, software engineering researchers have invested vast resources to understand and model the evolution of open source software and its communities for the past two decades. According to prior literature $[3,7]$, the OSS development largely depends on small groups

This work is licensed under a Creative Commons Attribution International 4.0 License. ESEC/FSE '20, November 8-13, 2020, Virtual Event, USA

(C) 2020 Copyright held by the owner/author(s).

ACM ISBN 978-1-4503-7043-1/20/11.

https://doi.org/10.1145/3368089.3418541 of developers, who undertake the most of the technical and social responsibility. They lead the amount and quality of contribution, and also positively impact their communities in various means.

Understanding the activities of these central developers in the OSS communities has particular importance for researchers and practitioners. With the advanced understanding, software engineering researchers may construct a set of principles to predict how developers influence the OSS project at various stages, and support their daily workflow for improved outcomes.

To understand and support these critical developers, especially the elite developers who own the administrative privileges for the project, first, I create an activity analysis framework and conduct two empirical studies to understand their impact on the community from the perspective of standalone project and OSS ecosystem. My results so far suggest that OSS projects are largely driven by the contributing activities of these elite developers. They make the majority of contributions in all activity categories. As the project grows over time, such as accepting external developers, these elite developers undergo role changes from code contributors to management positions in the community. However, while they shifting their focus to non-coding activities in later stages of the project, such change has a negative association with the project's technical outcomes in terms of productivity and quality. As many of the routine tasks can be automated with current technology, it leads to my research question: How can automation assist elite developers with their daily workflow?

Based on my results of elite developers' activities, I intend to integrate an automation toolset into the elites' daily workflow to reduce their efforts on some non-technical routine activities which may lower the technical outcomes of the project, and further implement and evaluate this tool with the activity analysis framework. The tool is designed as a plugin deployed into the GitHub environment. It leverages GiтHuB's event logs to monitor and summarizes developers' daily activities. The goal of this tool is to support elite developers making informed decisions on managing their efforts and finally improve the project's outcome and its sustainability.

Further, the toolset's evaluation is planned as an interview study at beginning on developers' usage and preference of automation, and following with a diary study in which participants apply the automation toolset. During the study, the tool monitors their activities and records project outcomes with the assistance of automation. Finally, elite developers' performance and feedback could yield meaningful implications for future OSS research.

\section{BACKGROUND}

Among the developer community, some "great designers" have a significantly positive influence on the software product and the 
Table 1: Multiple central roles a developer may play in OSS development.

\begin{tabular}{llll}
\hline $\begin{array}{l}\text { Central } \\
\text { Roles }\end{array}$ & $\begin{array}{l}\text { Research Context } \\
\text { and Scope }\end{array}$ & $\begin{array}{l}\text { Characteristics } \\
\text { for Identification }\end{array}$ & Definition \\
\hline $\begin{array}{l}\text { Core } \\
\text { Developer }\end{array}$ & $\begin{array}{l}\text { Community } \\
\text { Structure and } \\
\text { Hierarchy }\end{array}$ & $\begin{array}{l}\text { Amount of social and technical } \\
\text { contribution; Centrality in the social } \\
\text { and technical dependency network }\end{array}$ & $\begin{array}{l}\text { A group of developers who make substantial } \\
\text { contribution, and directing the development of } \\
\text { the project and community [5, 7]. } \\
\text { OSS contributors who make steady and frequent } \\
\text { technical contributions in contrast to one time } \\
\text { contributors [12]. }\end{array}$ \\
$\begin{array}{l}\text { Elite } \\
\text { Developer }\end{array}$ & $\begin{array}{l}\text { Contributing } \\
\text { Activity Patterns }\end{array}$ & Length and frequency of contribution \\
and Responsibility & $\begin{array}{l}\text { Holding the administrative privilege } \\
\text { and performing tasks that require such } \\
\text { privilege. }\end{array}$ & $\begin{array}{l}\text { A group of developers who hold the administrative } \\
\text { privilege in the project [11]. }\end{array}$ \\
\hline
\end{tabular}

performance of other developers [1,2]. These experienced expert developers not only produce high-quality source code and other technical artifacts, but also spend significant effort supporting other novice and inexperienced developers [9].

With the popularity of OSS development since the late 1990s, academic researchers have extensively investigated the activities of OSS developers. They found that developers contribute to OSS projects through various activities on code hosting platforms. More recently, the most popular code hosting site, GiтHuB, where streamlines the online workflows of developers [4], facilitates the collaboration among different projects with prominent data transparency. Developers apply various features embedded in the platform to support their social and technical activities in the software development. Besides contributing source code, they communicate with users, support external contributors, and delegate tasks to other team members [7, 11]. Their activities shape the project and its developer community in various means.

According to prior literature, the development of the OSS communities is driven by small groups of central developers [3, 7]. The interactions between these groups and other members in the communities form complex role-based relationships. As software engineering literature develops over the past two decades, various research constructs around central developers were postulated for focusing on specific research questions. In particular, different central roles were described (see Tab. 1). A central developer may belong to one or more (or all) of these roles. However, the roles are only meant to serve as a focus for specific research questions and issues in the context that a particular developer may be acting in. Particularly, I focus on classifying developers based on their privileges in this dissertation, and thus follow the notion of elite developers.

\section{APPROACH}

The automation that I seek to provide leverages GitHuB's event $\log$ as the major activity data source, and applies a set of statistic methods to provide analytics support to elite developers.

\subsection{Tool Design}

The proposed tool includes three major components, a data collector, an activity analyzer, and an automation toolset (see Fig. 1). The automation toolset mainly supports routine activities under the communicative and supportive categories. The selection of tools is suggested by prior my empirical studies on elite developers' activities at standalone project and ecosystem level, but yet to be determined via the following interview study with elite developers' usage and preference of automation.

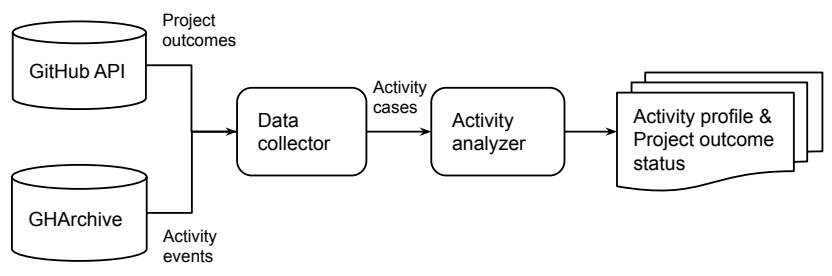

Figure 1: Data flow of the activity analysis framework.

Table 2: Definition of predefined activity categories.

\begin{tabular}{ll}
\hline Category & Definition \\
\hline Communicative & $\begin{array}{l}\text { Public and visible communication through } \\
\text { commenting features on issues, commit, and } \\
\text { project milestones. }\end{array}$ \\
\hline Organizational & $\begin{array}{l}\text { Managing the project community and delegating } \\
\text { tasks, such as assigning code reviewers, and } \\
\text { managing internal and external contributors. }\end{array}$ \\
\hline Supportive & $\begin{array}{l}\text { Non-coding activities in the collaborative OSS } \\
\text { development, including documentation and } \\
\text { versioning control. }\end{array}$ \\
\hline Typical & $\begin{array}{l}\text { Conventional coding task at the individual level, } \\
\text { such as submitting code reviews, commits and } \\
\text { pull requests. }\end{array}$ \\
\hline
\end{tabular}

The data collector collects the activity records and project outcome data from two major data sources including GHARCHIVE and GiтHuB. After raw event data is collected, I map atomic event types onto four predefined activity categories in a closed-card sorting process with two other researchers ( $0.77 \mathrm{Kappa})$. The categorization of activities follows a prior field study about developers' daily activities [9], but has been redefined under the context of software development with GitHus (see Tab. 2). Besides, the technical outcomes of the project are evaluated through four major indicators 
including the numbers of commits and bugs, bug cycle time, and bug fix rate $[10,11]$.

Table 3: Choices of data analysis methods.

\begin{tabular}{ll}
\hline Goal & Data Analysis Methods \\
\hline $\begin{array}{l}\text { Summarizing } \\
\text { activity profiles }\end{array}$ & Descriptive analysis \\
\hline $\begin{array}{l}\text { Identifying trend of } \\
\text { contributing efforts }\end{array}$ & $\begin{array}{l}\text { Descriptive statistics, } \\
\text { One way ANOVA }\end{array}$ \\
\hline $\begin{array}{l}\text { Testing relationships } \\
\text { between efforts and } \\
\text { project outcomes }\end{array}$ & $\begin{array}{l}\text { Fixed effects Panel } \\
\text { Regressions }\end{array}$ \\
\hline
\end{tabular}

The activity analyzer establishes longitudinal activity profiles according to developers' efforts on each type of activities, and tests the relationship between their efforts and project outcomes. A summary of my data analysis methods is included in Tab. 3. To generate a complete description of their activity profiles, I compare their activity with non-elite members in the project. Also, the growth rate of elite developers' effort in each type of activities is computed and then compared through the one-way ANOVA test. Thus, I could identify the trend of how developer allocate her efforts as each project growing over time, and test the difference between each type of activities. Finally, to test the relationship between their efforts and project outcomes (in terms of productivity and quality [10]), the fixed effects panel regression is applied. The selection of the econometric method is based on the multi-sectional and longitudinal nature of the activity dataset.

\subsection{Evaluation}

The evaluation plan is to conduct interviews and diary studies with elite developer participants. The evaluation has three stages In the beginning, elite developer participants will be interviewed about their workload on GiтHuB, usage and preference of automation assistance, and challenges about managing their online efforts. Therefore, I am able to prepare and integrate an automation toolset with the activity analysis framework to assist their non-technical tasks. In the second phase, I deploy the tool with their GiтHuB environment to monitor their activities, and provide analyses on the impact of their contributing activities as well as project outcome summary up to six weeks. At the end of the evaluation, participants are invited to an exit interview for answering open-ended questions about the usability and usefulness of the tool, and whether the tool is beneficial to managing their routine.

\section{RESULTS TO DATE}

The data analysis components have been applied in two empirical studies on elite developers' activities. My results so far suggest the significant importance of elite developers in the open source community. I found that compared with numerous non-elite developers, elite developers make a significant number of contributions. Further, these elite developers are moving to the management positions from major code contributors, and such transition increases their non-coding responsibilities. As this transition leads to more efforts allocated on the supportive and communicative activities, it

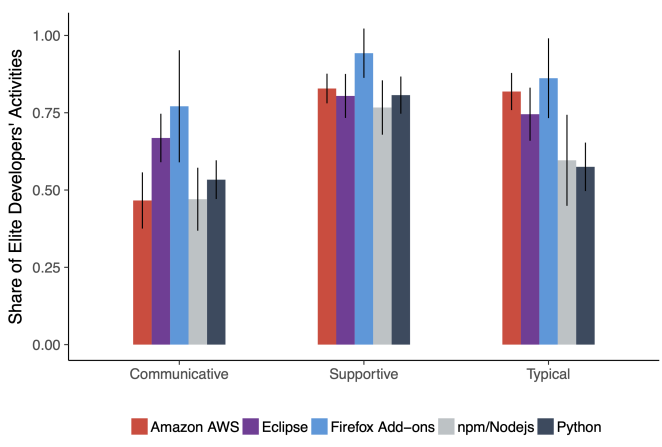

Figure 2: Ratios of elite developers' contributing activities in sampled ecosystems.

Table 4: Panel regression models for project outcomes.

\begin{tabular}{|c|c|c|c|c|}
\hline & \multicolumn{2}{|c|}{ Productivity } & \multicolumn{2}{|c|}{ Quality } \\
\hline & $\begin{array}{l}\text { NewCommit } \\
(S E)\end{array}$ & $\begin{array}{l}\text { BugCycleTime } \\
(S E)\end{array}$ & $\begin{array}{l}\text { NewBug } \\
(S E)\end{array}$ & $\begin{array}{l}\text { BugFixRate } \\
(S E)\end{array}$ \\
\hline$\overline{S-\text { Com }_{\text {im }}}$ & $\begin{array}{l}-155.96^{* *} \\
(49.20)\end{array}$ & $\begin{array}{l}-170.71 \\
(143.96)\end{array}$ & $\begin{array}{l}4.13 \\
(5.26)\end{array}$ & $\begin{array}{l}-2.24^{* * *} \\
(0.35)\end{array}$ \\
\hline$\overline{S-\mathrm{Org}_{i m}}$ & $\begin{array}{l}1.38 \\
(121.25)\end{array}$ & $\begin{array}{l}-148.61^{*} \\
(54.65)\end{array}$ & $\begin{array}{l}50.13^{* * *} \\
(12.97)\end{array}$ & $\begin{array}{l}-0.63 \\
(0.87)\end{array}$ \\
\hline$\overline{S-S u p_{i m}}$ & $\begin{array}{l}-138.21^{* * *} \\
(35.52)\end{array}$ & $\begin{array}{l}473.60^{* * *} \\
(103.94)\end{array}$ & $\begin{array}{l}18.31^{* * *} \\
(3.80)\end{array}$ & $\begin{array}{l}1.12^{* * *} \\
(0.26)\end{array}$ \\
\hline $\begin{array}{l}\text { Unobserved time } \\
\text {-invariant effects } \\
\left(\alpha_{i}\right)^{\mathrm{I}}\end{array}$ & $--^{* * *}$ &.$-^{* * *}$ &.$-^{* * *}$ & $--^{* * *}$ \\
\hline Multiple $R^{2}$ & 0.884 & 0.745 & 0.857 & 0.649 \\
\hline Adj. Multiple $R^{2}$ & 0.880 & 0.736 & 0.853 & 0.637 \\
\hline$F^{\dagger}$ & $231.10^{* * *}$ & $88.35^{* * *}$ & $186.2^{* * *}$ & $56.09^{* * *}$ \\
\hline
\end{tabular}

has a negative relationship with the project's technical outcomes in terms of productivity and quality. Finally, I have identified some OSS ecosystem-specific effects on elite developers' activities with the ecosystem's domain. For instance, the Python ecosystem is usually developed by interdisciplinary teams, and performing organizational activities exhibits strong effects on its technical outcomes.

By comparing elite developers' activities with the non-elites', I find that the community is primarily driven by elite developers' contributing activities. Elite developers are small groups among all contributors, but they still conduct most of the projects' contributing activities. Except for the communicative activities, elite developers perform over $50 \%$ of the activities in the other three activities categories. Particularly, when comparing the amount of contributing workload at individual average, there are orders of magnitude difference between elite and non-elite developers. Noticeably, through comparing elite developers' activity profiles across various software ecosystems, the type of projects also correlates with the ratio of elite developers' workload. For example, elite developers in smaller add-on projects usually have a heavier workload than others (see Fig. 2, and note that: organization activities can only be performed by elite developers who hold the privileges, so we do not present their shares here). 
Further, according to the longitudinal observation, there is a significant role transition of elite developers. Through computing the growth rate of each type of activities and conducting one-way ANOVA tests on these rates, the collected results show significant differences between the three categories (the organizational activities are excluded due to the division of zero error while calculating growth rate). The post-hoc analysis is conducted to identify the differences, and the results of Tukey's HSD test indicates that the growth rates of typical activities are significantly lower than the growth rates of the other two (Typ. vs. Com.: $p=0.002$, Typ. vs. Sup.: $p=0.002$ ). These results suggest that elite developers start OSS project majorly as code contributors who build the project's infrastructure. However, as the project and its community growing, they reduce their contribution in typical coding but conduct more activities in supporting and communicating with other developers. Especially when more external developers joining into the project and submitting their technical contributions, elite developers have to spend more efforts into these non-coding responsibilities.

The role transition and change of working focuses have a negative impact on the project outcomes. To identify the relationship between the elite developer's effort and project outcomes, I empirically test them by constructing a series of regression models. I test the relationship between elite developers' efforts in three non-coding activity categories and four outcome indicators, including two project productivity and two code-base quality indicators. These indicators are widely used in prior literature to assess the project outcomes for those OSS projects adopted the continuous integration model $[6,8,10]$. The results of the panel regression show various strengths on four indicators (see Tab. 4), but in general, elite developers allocating efforts on non-typical activities are negatively associated with project outcomes.

\section{CONTRIBUTION}

This dissertation first purposes a fresh approach to investigating an important group of developers and employs the privilege system in the collaboration platform to classify developers. It develops a categorization of activities to frame developers' activity profiles and investigate developers' effort allocation, which considers developers' full-spectrum online activities. It further establishes the relationships between developers' effort allocation and OSS project's technical outcomes.

Second, it deepens our understanding of the OSS development and unveils that the elite groups actively drive the OSS community's development. The results suggest the elites' critical roles in the OSS development. They have to engage in most of the projects' activities, and their technical contributions only account for a small proportion. Besides, elite developers have contributed significantly, especially in smaller and less popular projects. Their heavy social and technical responsibilities increase as more external developers contributing to the project, and leaving heavy burdens on elite developers are harmful to the sustainability of the OSS development. Further, my studies also identify the inevitable role transition of elite developers and the negative impact of such a transition.

Third, this dissertation provides practical insights into effectively managing elite developers' efforts with a proposed automation toolset that targets at assisting elite developer's non-technical routine activities. With the assistance of automation, I aim to minimize developer's effort on routine tasks. The proposed tool also increases the developers' awareness of her activity in the project and suggests how her activities may impact the project. It presents how developers' workflow changes from a longitudinal perspective, and it helps developers project their career path.

\section{CONCLUSION AND FUTURE WORK}

My Ph.D. studies investigate and model how elite developers drive the development of the OSS communities from multiple perspectives. I take a fresh approach to investigate elite developers' activities while considering the allocation of efforts on four activity categories. My studies present elite developers' activity profiles extract from fine-grained event data, including their social and technical contributions to the community. Further, through these mix-methods studies, I summarize how automation can support elite developers' daily workflow.

As I am completing my two empirical studies of elite developers workflows, the remaining work focuses on the tool implementation and evaluation. Elite developers' preference of automation support should be concluded from a following interview, and then technical decisions need to be made about how to implement and deploy the tool in the GiтHuв environment. The preparation of the user study and also the recruitment of participants are expected to be finished by February 2021. The user study and data analysis may take the following four months, and remaining months are for completing the writing of the dissertation.

\section{REFERENCES}

[1] Barry W. Boehm. 1987. Improving software productivity. Computer 9 (1987), 43-57.

[2] F Brooks and HJ Kugler. 1987. No silver bullet. April.

[3] Kevin Crowston, Kangning Wei, James Howison, and Andrea Wiggins. 2008. Free/Libre open-source software development: What we know and what we do not know. ACM Computing Surveys (CSUR) 44, 2 (2008), 1-35.

[4] Laura Dabbish, Colleen Stuart, Jason Tsay, and Jim Herbsleb. 2012. Social coding in GitHub: transparency and collaboration in an open software repository. In Proceedings of the ACM 2012 conference on computer supported cooperative work. 1277-1286.

[5] Mitchell Joblin, Sven Apel, Claus Hunsen, and Wolfgang Mauerer. 2017. Classifying developers into core and peripheral: An empirical study on count and network metrics. In 2017 IEEE/ACM 39th International Conference on Software Engineering (ICSE). IEEE, 164-174.

[6] Foutse Khomh, Tejinder Dhaliwal, Ying Zou, and Bram Adams. 2012. Do faster releases improve software quality? an empirical case study of mozilla firefox. In 2012 9th IEEE Working Conference on Mining Software Repositories (MSR). IEEE, 179-188.

[7] Audris Mockus, Roy T Fielding, and James D Herbsleb. 2002. Two case studies of open source software development: Apache and Mozilla. ACM Transactions on Software Engineering and Methodology (TOSEM) 11, 3 (2002), 309-346.

[8] Baishakhi Ray, Daryl Posnett, Vladimir Filkov, and Premkumar Devanbu. 2014. A large scale study of programming languages and code quality in github. In Proceedings of the 22nd ACM SIGSOFT International Symposium on Foundations of Software Engineering. 155-165.

[9] Sabine Sonnentag. 1995. Excellent software professionals: Experience, work activities, and perception by peers. Behaviour \& Information Technology 14, 5 (1995), 289-299.

[10] Bogdan Vasilescu, Yue Yu, Huaimin Wang, Premkumar Devanbu, and Vladimir Filkov. 2015. Quality and productivity outcomes relating to continuous integration in GitHub. In Proceedings of the 2015 10th Foint Meeting on Foundations of Software Engineering. 805-816.

[11] Zhendong Wang, Yang Feng, Yi Wang, James A Jones, and David Redmiles. 2020. Unveiling Elite Developers' Activities in Open Source Projects. ACM Transactions on Software Engineering and Methodology (TOSEM) 29, 3 (2020), 1-35.

[12] Minghui Zhou and Audris Mockus. 2012. What make long term contributors: Willingness and opportunity in OSS community. In 2012 34th International Conference on Software Engineering (ICSE). IEEE, 518-528. 